\title{
A review of implantable pulsatile blood pumps: engineering perspectives
}

Tingting $\mathrm{Wu}^{1}$, Ashraf W Khir ${ }^{2}$, Maximilian Kütting ${ }^{3}$, Xinli Du², Hao Linn ${ }^{1}$, Yuxin Zhu ${ }^{1}$, Po-Lin Hsu ${ }^{1 *}$

1. Artificial Organ Technology Laboratory, Biomanufacturing Centre, School of Mechanical and Electrical Engineering, Soochow University, Suzhou, China

2. Brunel Institute for Bioengineering, Brunel University London, Uxbridge, UK 3.R\&D, NVT GmbH, Germany

\begin{abstract}
It has been reported that long-term use of continuous-flow mechanical circulatory support devices (CF-MCSDs) may induce complications associated with diminished pulsatility. Pulsatile-flow mechanical circulatory support devices (PF-MCSDs) have the potential of overcoming these shortcomings with the advance of technology. In order to promote indepth understanding of PF-MCSD technology and thus encourage future MCSD innovations, engineering perspectives of PF-MCSD systems, including mechanical designs, drive mechanisms, working principles and implantation strategies, are reviewed in this article. Some emerging designs of PF-MCSDs are introduced and possible elements for next generation PF-MCSDs are identified.
\end{abstract}

Keywords: Mechanical circulatory support device; Pulsatile blood pump; Pumping mechanism; Blood-contact; Ventricular assist devices; Total artificial heart 


\section{Introduction}

Six and half million adults have been diagnosed with heart failure (HF) with 960,000 new cases annually in the US alone ${ }^{1}$. The 1-year and 5-year mortality of end-stage HF is $30 \%$ and $50 \%$, respectively ${ }^{2}$. Heart transplantation remains the gold standard to treat endstage HF (survival of 9.5 years) but available donors are far too few to meet the need. From September 1987 to December 2012, over 40,000 patients were waiting for heart transplants with a survival of 2.3 years; only about 27,000 received donor hearts ${ }^{1}$. Thanks to the smaller size, simplicity, and reliability of the rotary blood pump technology, CFMCSDs), known as the second and the third-generation blood pumps, have shown a much improved survival ( $81 \%$ in 1 year) and patient outcomes compared to the first generation predecessors which generated pulsatile flow ${ }^{3-5}$. However, the operation of CF-VADs reduces the intrinsic pulsatility in the circulatory system, which could be linked to a lower cardiac recovery rate $(<2 \%)$ than with pulsatile-flow VADs (PF-VADs) and complications including aortic insufficiency (AI), thromboembolic events, impaired microcirculation, right heart failure (RHF), and acquired von Willebrand syndrome (AvWS) and bleeding events ${ }^{6-14}$.

To date, pulsatile flow seems to provide more long-term benefits over purely continuous flow. Attempt has been made to generate pulsatile flow by incorporating speed modulation in the CF-MCSDs ${ }^{6,15}$. More research is required to examine that if these 
control algorithms are capable of providing adequate pulsatility ${ }^{8}$. Furthermore, there is no guarantee that with the frequent speed change of the impeller, hemocompatability will remain the same with current pump designs.

Even though the issues related to the design of the first-generation pulsatile devices have effectively prevented their use in clinical arena, the physiological benefits are still promising. It is worthy to take a retrospective overview on current engineering foundation, the design principle, and related complication in order to revisit the need and stimulate next-generation design.

The devices included in this review are categorized into four groups - left ventricular assist devices (LVADs) before 2008, LVADs after 2008, counter-pulsation devices, and total artificial hearts (TAHs). The year 2008 was used in consistence with introduction of the first FDA approved continuous flow device, emphasizing the transformation of the pump designers' strategy with the accumulating experience. The technical specifications are summarized in Table 1 and the focus of the following paragraphs is to describe the engineering perspectives.

\section{Devices review}

Generally speaking a PF-MCSD consists of a volume-displacement pump unit, a control unit, and a powering unit. The pump units differ from each other in the driving mechanism - mechanically, pneumatically or hydraulically. The pump units are controlled by either 
controllers or consoles depend on patients' mobility demand. Power is supplied by percutaneous driveline (PD) connecting to the external battery or other power sources or transcutaneous energy transfer (TET) systems. The overview of the mechanical specifications is summarized in table 1, while following paragraph focus on the distinguished design, pumping mechanism and clinical performance of each pump.

Table 1. Parameters of the implantable PF-MCSD

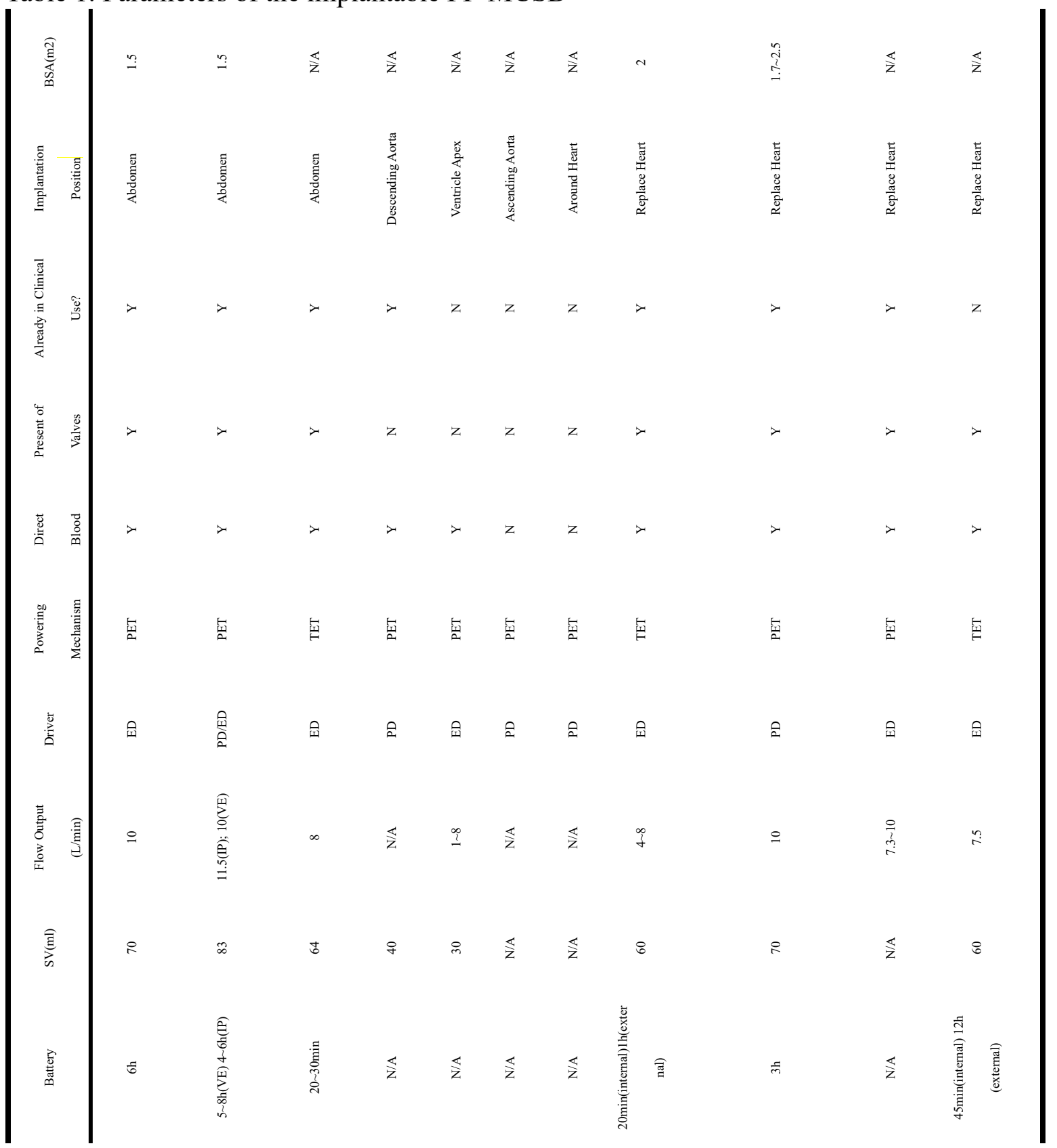




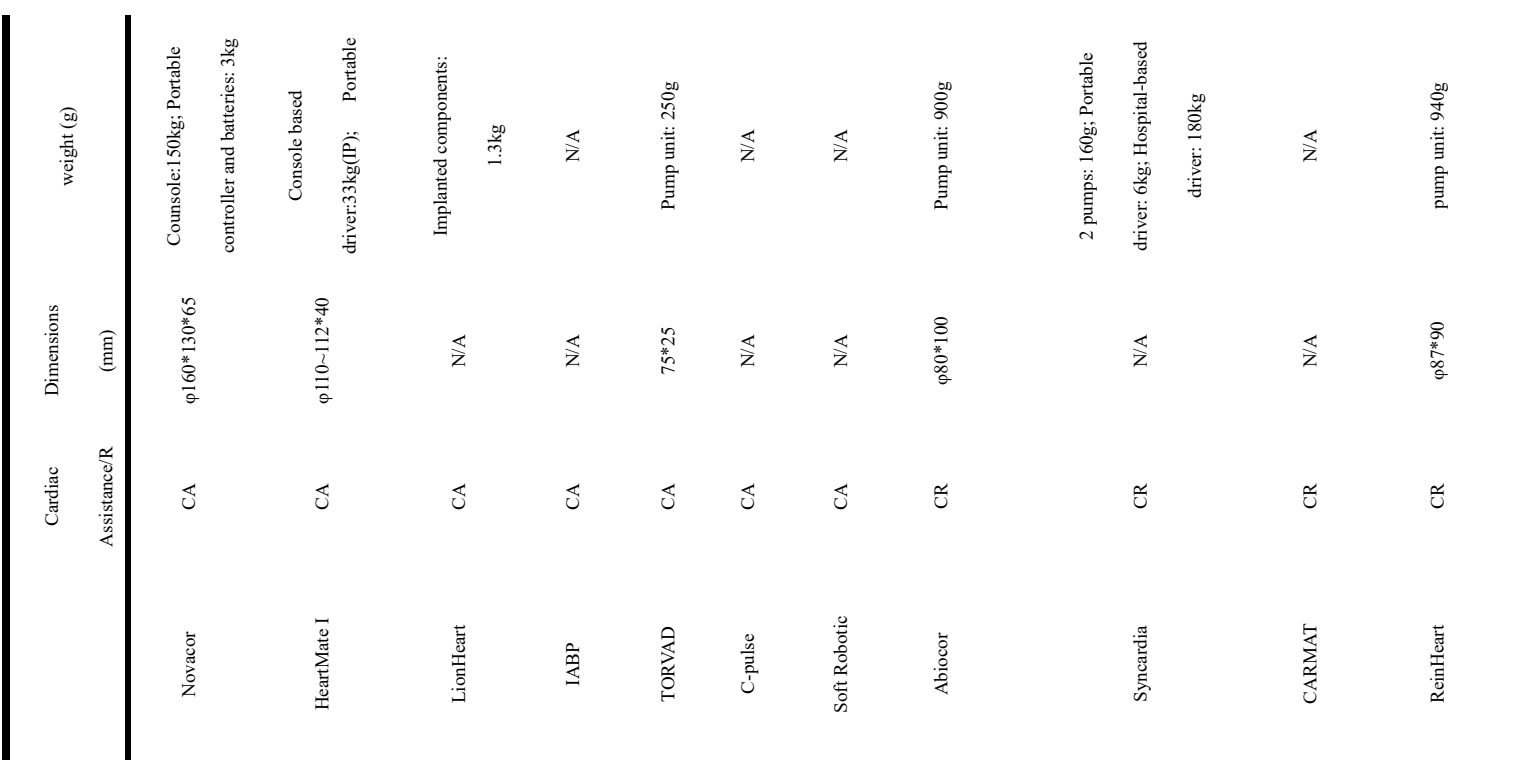

N/A: Not Applicable; CA: Cardiac Assistance; CR: Cardiac Replace; SV: Stroke Volume; ED: Electriclly Driven; PD: Pneumatically Driven; PET: Percutaneous energy transmission; TET: Transcutaneou energy transmission.

\section{LVADs up to 2008 (Not in clinical use)}

Novacor, HeartMate I (Fig.1), LionHeart (Fig.2) systems were devices that had been developed before 2008, among them Novacor was the first FDA approved mechanical circulatory assist system. These three systems included volume-displacement pump units, drive units, controller and batteries, detailed parameters were included in table $1^{16-20}$. All three pump units contained blood sacs, which were either driven electrically (deformed by pusher plates) or pneumatically ${ }^{16,19-21}$. Blood contacting surface of HeartMate I was textured with sintered titanium microspheres on the titanium surfaces and fibrillar textured surface on the sac diaphragm ${ }^{22}$. Instead of using percutaneous driveline (PD), LionHeart was the first VAD incorporating Transcutaneous Energy Transmission (TET) system $^{23}$.

These pulsatile pumps operated in synchronize with native heart ${ }^{24,25}$. However, the big 
volume of those pumps required invasive implantation surgeries and patients with big body surface area $\left(>1.5 \mathrm{~m}^{2}\right)^{21,26,27}$. Infection around the PD (48\% readmission) and embolic accident around the inflow valve area (2 out of 8 paatients, from Feburary 1992 to April 1994) were the main complications with the Novacor device ${ }^{16,28}$. Device malfunction rate of HeartMate I decreased from $4.6 \%$ in 1995 to $1.1 \%$ in 1999 , due to device improvements ${ }^{29}$. With all the efforts LionHeart had made, $37 \%$ decrease in sepsis incidence and $26 \%$ decrease in death attributed to sepsis were achieved. However, it also brought in the device-related infections (35\%), including 7 pocket infections and 1 compliance chamber infection out of 10 events, with Dincreased invasiveness and complexity of implantation ${ }^{30-32}$.

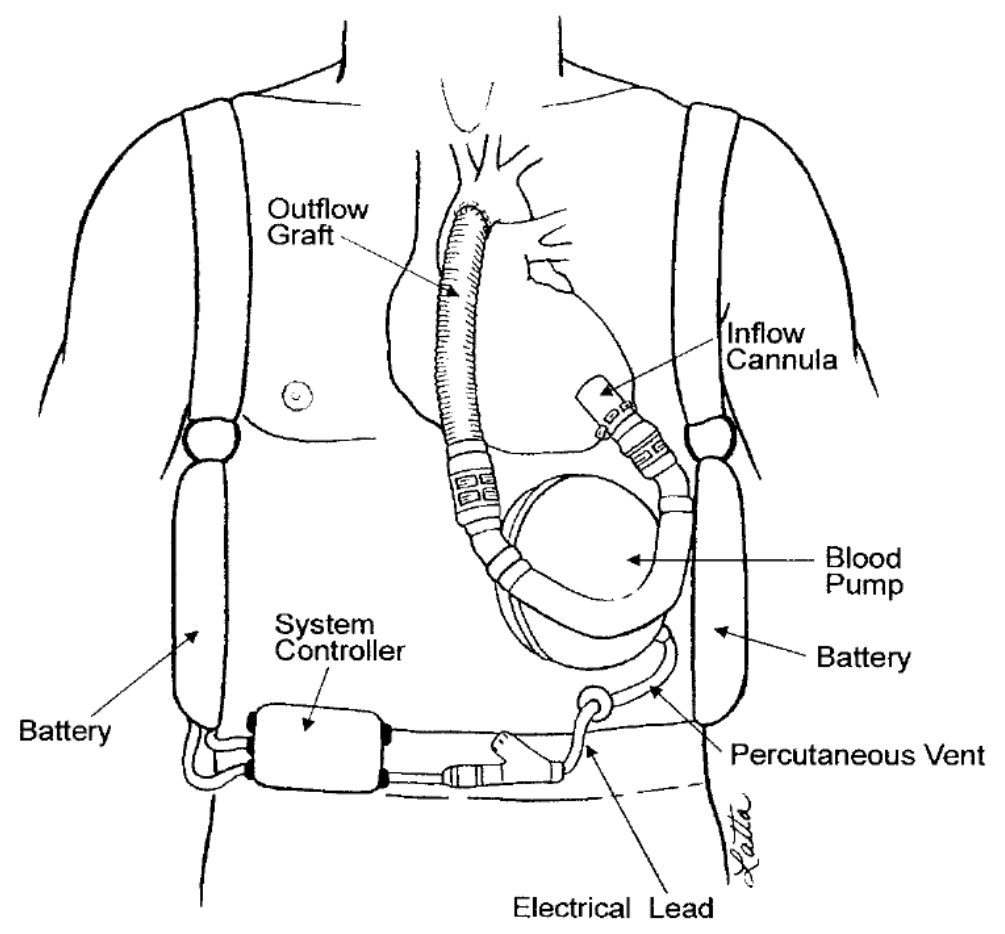

Fig.1 HeartMate VE system 


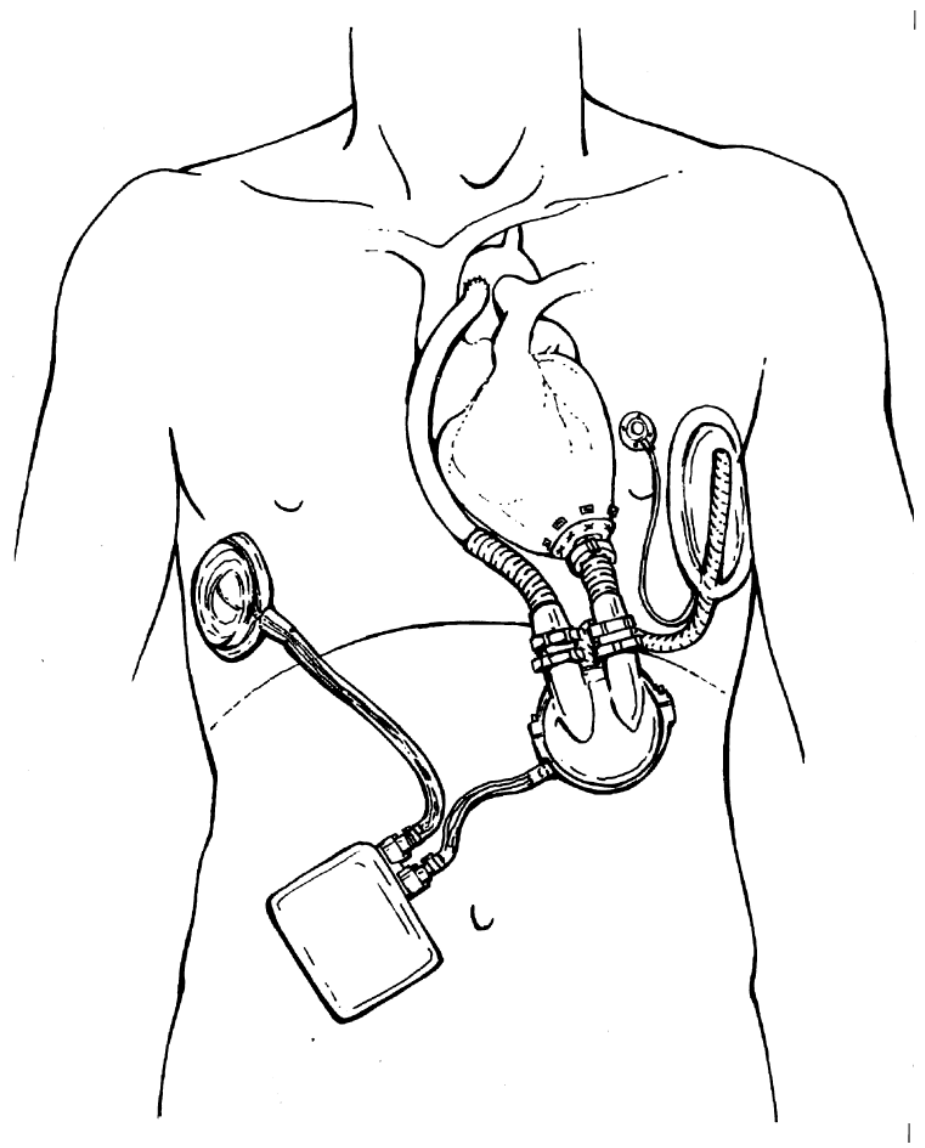

Fig.2 LionHeart system

\section{LVADs after 2008}

\section{$\underline{\text { TORVAD (research device, not in clinical use) }}$}

TORVAD device is a valveless pulsatile blood pump with two rotary pistons, eliminating the thromboembolic risk around the artificial valves. The innovative driving/pumping mechanism involves rotating one piston around a toroidal-shaped chamber while maintaining the other piston between inlet and outlet ports. These two pistons exchange roles when one stroke circle finishes, facilitating unidirectional flow without the presence of one-way valves. Normally, the TORVAD device synchronizes 
with native cardiac cycle, providing $30 \mathrm{ml}$ output per stroke. When under asynchronous operation, the device is able to provide 1 to $8 \mathrm{~L} / \mathrm{min}$ flow output to suit the patient's demand. This unique working principle allows smaller size, allowing for the pre- or intraperitoneal implantation ${ }^{33-35}$. Superior hemocompatibility was reported.

\section{Soft Robotic Sleeve (research device, not in clinical use)}

Soft Robotic Sleeve is a non-blood-contacting pump to be implanted in the pericardial space and wrapped around the heart. This device contains soft pneumatically driven artificial muscles arranged in helical and circumferential patterns same as the outer two layers of heart muscle fibers, and silicon sheets that incorporate the actuators. Muscle patterns (helical or circumferential) and part of the actuator (right or left) can be controlled independently to provide customized assistance for various heart diseases.

The soft robotic sleeve is designed to synchronize with the native cardiac cycle during which the device contracts and twists in systole and relaxes during diastole. Since there is no foreign material in contact with blood, the need for anticoagulation is eliminated for this device. The in-vivo results proved an increased ejection output in the porcine cadaver hearts and reestablishing cardiac output (from 45\% to 97\%) in an acute porcine asystole $\operatorname{model}^{36}$. 


\section{Counter-pulsation devices}

\section{Intra-Aortic Balloon Pump}

Intra-aortic balloon pump is the first-in-line resuscitation therapy and the most commonly used left ventricular assist devices for its low cost and ease of insertion/removal/replacement 37,38 . It contains intra-aortic balloon, percutaneous driveline, console, and helium tank. The intra-aortic balloon is a dual-lumen catheter - the inner lumen connected to a pressure sensor and the outer lumen to a gas supply. A standard balloon has a volume of $40 \mathrm{ml}^{39}$ and mounted on a 7 or 8 -Fr catheter. The balloon inflates during native heart diastole, after the aortic valve closure, to increase the coronary perfusion. Then deflates quickly in early systole, prior to the opening of the aortic valve, to create a temporary vacuum in the descending aorta which reduces afterload thereby helps left ventricle unloading ${ }^{40,41}$.

The pump is implanted through arterial access to the descending aorta. The balloon should be placed above the renal artery and about $2 \mathrm{~cm}$ below the subclavian artery. Its insertion site requires patients to remain supine position during the whole therapy, leading to circumscribed mobility ${ }^{42}$. The effect of the balloon shape was investigated by Kolyva et al, due to the diminished benefits when the patient is nursed in the semirecumbent position. A lanceolate shaped IAB with $2 / 3$ conical segments was less affected by angulation and even exceeded the performance compared to a cylindrical IAB during inflation $^{43}$.

IABP SHOCK I trial demonstrate no significant effect on 30 day mortality, this results 
was further strengthened by SHOCK II trial with only slight improvement in 12 month mortality. The minor hemodynamic effects could be attributed to the similarity in mortality between IABP and control group for both SHOCK trials. What's also similar in both trials was about $90 \%$ of survivors were in NYHA class I/II ${ }^{44,45}$.

\section{NuPulseCV intravascular ventricular assist system (iVAS) (research device, not in}

\section{clinical use)}

NuPulseCV iVAS (Fig.3) may be considered as a long-term version of IABP to provide partial yet adjustable support for advanced HF. The design is intended to combine features of IABP and LVAD to promote minimally invasive approach but still allow patient mobility. The system consists a 50 c.c. balloon pump, skin interface device (SID), three subcutaneous electrodes and a portable driver. The counter-pulsating balloon pump is to be placed in the descending aorta through the subclavian artery ${ }^{46}$. The SID is an electromechanical and pneumatic conduit with a chimney that allows for shuttling of air between the pump and external driver and communication of the captured ECG signals that are transmitted to the driver from 3 subcutaneous electrodes ${ }^{47}$.

In the first-in-human trial, 13 out of 14 patients were ambulatory within 24 hours and successfully transplanted after. One patient needed escalation of support and received Impella 5.0 then CentriMag blood pump for ECMO before transplanted. There was no pump thrombosis, stroke, and gastrointestinal bleeding in this single center study ${ }^{47}$. 


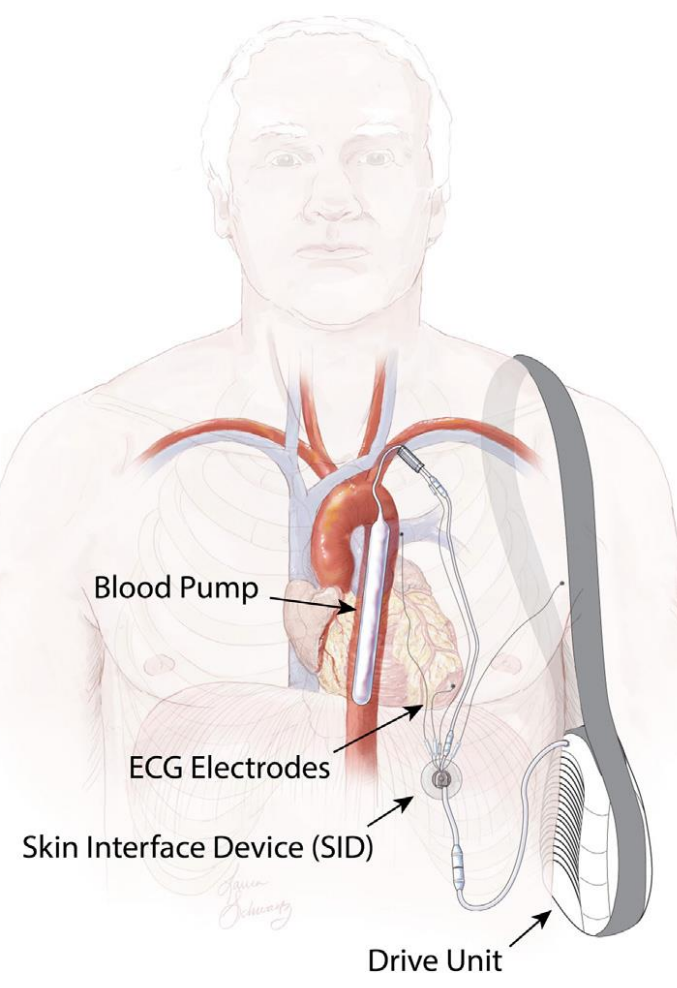

Fig.3 NuPulseCV iVAS

\section{The Kantrowitz CardioVAD (KCV) (research device, not in clinical use)}

Another attempt for long-term pulsatile balloon pump is the Kantrowitz CardioVAD, which utilizes extra-aortic placement and provides partial support for HF patient not qualified for transplant ${ }^{48,49}$. The KCV consists of a $60 \mathrm{cc}$ Cardioplus pump, percutaneous driveline and a portable controller. The 60 c.c., super lightweight balloon pump $(<0.03$ $\mathrm{kg}$ ) is to be attached on the descending aorta via surgical procedure. The invasive implantation potentially increases the risk of morbidity and mortality; however, the nonblood-contacting design of the KCV does allow the device to be turned on/off as needed without increasing the risk of thromboembolism and does not require any anticoagulation 49,50 


\section{C-Pulse (Not in clinical use)}

C-pulse(Fig.4) is also an extra-aortic balloon pump ${ }^{51}$. The C-pulse system comprises an extra-aortic cuff/balloon, sensor heads, a percutaneous driveline, a driver and batteries 52. The pre-shaped balloon is wrapped around the ascending aorta right above the sintubular junction by an aortic cuff, and connected to the gas tank with a percutaneous driveline. With the same inflation/deflation mechanism as of the IABP, the C-pulse increases left ventricle diastole pressure and coronary perfusion during diastole and reduces ventricular afterload during systole. Nevertheless, like other non-bloodcontacting device, the C-Pulse system eliminates the requirement for anticoagulation, potentially reducing the risk of bleeding and device-related thrombosis.

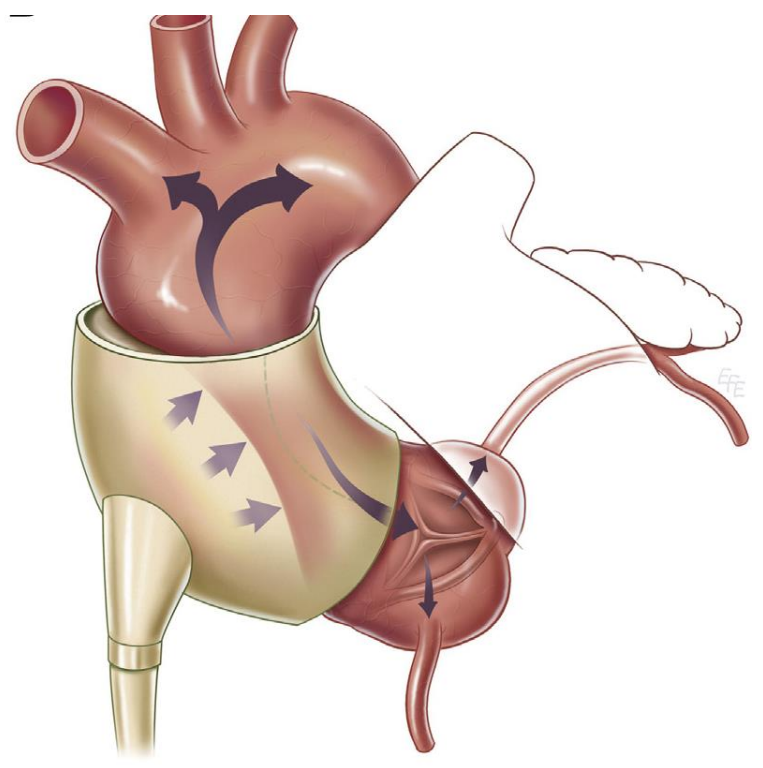

Fig.4 C-pulse pump

\section{Symphony (research device, not in clinical use)}

Symphony is a valveless pneumatically driven pump placed in the pacemaker pocket, 
eliminating the need of open chest surgery and providing partial cardiac support ${ }^{48}$. The Symphony device consists of a 30 c.c. volume displacement pump body made of polyurethane membrane, a console, an electrocardiogram leads, and percutaneous pneumatic driveline ${ }^{49,53}$. The pump body is to be placed in the pacemaker-like pocket in the infraclavicular fossa of the right side of the patient and connected to the subclavian artery with a single short graft $48,53,54$.

The counterpulsation of the pump was controlled by ECG signals ${ }^{53}$. The Symphony, in comparison to a 40-mL IABP, was capable of providing higher overall coronary, carotid, and aortic flows and eliminating retrograde flow ${ }^{11} 55$.

\section{TAHs}

\section{$\underline{\text { Abiocor TAH }}$}

Abiocor is the first available (approved as humanitarian device) electrohydraulic driven TAH, and the world's first fully implantable artificial heart with a TET system ${ }^{56}$. The external coil can be connected to the bedside console or the external batteries ${ }^{57}$. The external battery could provide approximately 1 hour operation, and the internal battery up to 20 minutes ${ }^{58,59}$. The Abiocor pump unit consists of two chambers that replace the position and function of two native ventricles. In between the two chambers is a hydraulic pump which displaces the blood sac membrane by actuating fluid ${ }^{56}$. An additional atrium chamber is connected to the left chamber to address the flow imbalance between left and right ventricles. A compensating chamber is separated from the atrium chamber by 
flexible diaphragm and connects to the right chamber. When left chamber is in the diastole, the hydraulic fluid flows to the right chamber and the compensating chamber, exerting less volume displacement than that in the left chamber systole. The process is reversed during left chamber systole ${ }^{60}$.

Implantation of the Abiocor system required highly invasive thoracotomy in patients with $\mathrm{BSA} \geq 2 \mathrm{~m}^{2}$. Two out of 14 patients died during the surgical procedure and the longest survival of the remaining patients was 512 days ${ }^{61-63}$. Multiple complications were reported, including 9 cerebrovascular accidents, 11 non-device-related infections, 2 device failure led death, 3 multiple organ failure, and 4 device related infections ${ }^{62-64}$.

\section{$\underline{\text { Syncardia CardioWest }}{ }^{T M}$ TAH}

Syncardia TAH (Fig. 5) is the very first mechanical circulatory support device used as destination therapy ${ }^{65}$. Two pneumatically driven pump units (approximately $160 \mathrm{~g}$ each), which differs in the integrated valves, are connected to the atria through velcro after excision of the native ventricles ${ }^{66} 67$. The external driver, connecting to the pump units via a pneumatic driveline, comes in hospital-based (about $180 \mathrm{~kg}$ ) and portable discharge (about $6 \mathrm{~kg}$ ) versions ${ }^{66,68}$. The portable driver can supply 3-hour operation without connecting to the main ${ }^{69}$. 


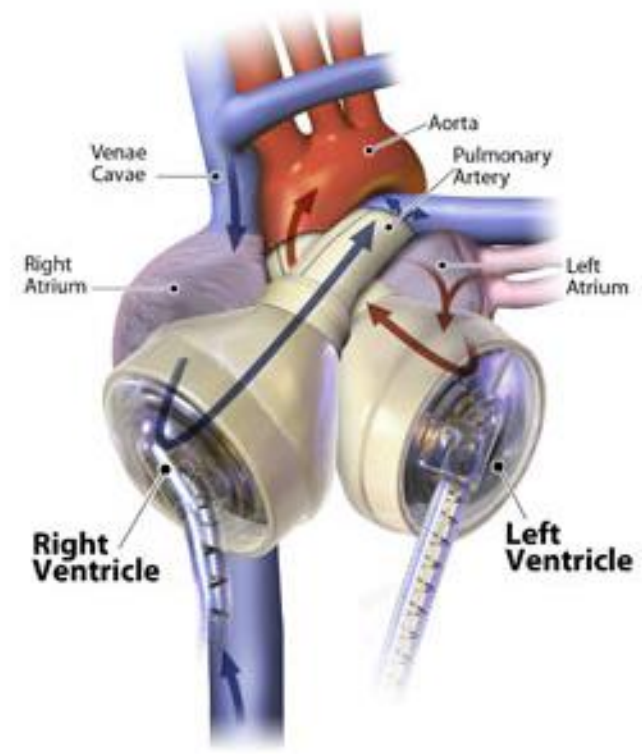

\section{Total Artificial Heart}

Fig. 5 Syncardia TAH

During cardiac systole, the driver system pushes air into the artificial ventricles through the pneumatic drivelines, and forces diaphragms deform towards the superior aspect of the blood chamber, ejecting blood out of the chamber. During cardiac diastole, the driver system sucks the air out, and creates temporary vacuum to help the blood chamber get filled.

Unfortunately, the $70 \mathrm{ml}$ stroke volume of each ventricles still limits the device use in patients with $\mathrm{BSA} \geq 1.7 \mathrm{~m}^{270}$. A multi-center study demonstrated a $79 \%$ bridged to transplant rate of the patients who received Syncardia TAH with an overall 1-year, 5-year, and 8 -year survival of $70 \%, 50 \%$, and $45 \%$, respectively 71,72 . Infection and hemocompatibility related complications were significant with the Syncardia TAH - 53\% systemic infections, $27 \%$ driveline infections, $19 \%$ thromboembolic events and $14 \%$ 
hemorrhagic events were reported in 47 patients $^{73}$.

\section{$\underline{\text { CARMAT TAH }}$}

Similar to the Abiocor TAH, the CARMAT TAH is also an electrohydraulic driven pulsatile blood pump. The CARMAT TAH differ in its blood contacting surfaces consisting of bovine pericardial tissue and expanded polytetrafluorethylene (ePTFE). The CARMAT pump is separated into a left ventricle and a right ventricle; each ventricle has a blood chamber and a hydraulic chamber by hybrid membrane. The silicone fluid driven by two electro-hydraulic pumps, exerting forces on the membrane and thus displacing blood out of the blood chamber into circulation. A short-term animal study demonstrated the ability of CARMAT pump to provide 7.3 to $10 \mathrm{~L} /$ min flow output $^{74}$. Like any other TAHs, CARMAT pump has four valves navigating blood flow, a percutaneous driveline supplying power and controlling pump unit ${ }^{70}$.

An intelligent control algorithm enables the device to response to preload and afterload spontaneously. Together with the use of prosthetic materials, these features gives more potential for a bio-compatible TAH device ${ }^{75}$. However, the integrated electronics and microprocessor in the implanted pump result in a bigger size $(>750 \mathrm{ml})$ which fits in $86 \%$ of male patients and only $14 \%$ of female patients ${ }^{70}$.

\section{$\underline{\text { ReinHeart TAH }}$}

ReinHeart is a totally implantable TAH with a TET system and is driven completely electrically by a linear motor, eliminating complex mechanical bearings and gears. Both 
blood chambers of the pump unit have $60 \mathrm{ml}$ stroke volume and can provide up to 7.5 $\mathrm{L} /$ min flow output $^{76,77}$.

The main aspect that distinguishes the ReinHeart TAH from other TAHs is the passively filling pump unit (Fig.6). The slider of the linear motor is connected to two pusher plates that exert forces on the blood chambers. Yet the pusher plates are separated from the blood chambers, which allow the blood chambers to fill passively during diastole, eliminating the need for preload detecting pressure sensors ${ }^{76}$. A compliance chamber, connected to the pump unit by a tube, cuts down pressure peaks and prevents suction events ${ }^{77,78}$. The internal controller controls the pump unit and compliance chamber and provides system status information. Furthermore, a control algorithm in response to preload and afterload was developed to address the imbalance between pulmonary circulation and system circulation ${ }^{79}$. Therefore, the ReinHeart device could provide customized assist for different physiological conditions.

The TET system allows the coil misalignment of $30 \mathrm{~mm}$, potentially increasing the usability in daily care ${ }^{76}$. The two external packable batteries can provide up to 12 hours operation through the TET system and the internal battery can supply 45 mins operation in case of the TET system's failure ${ }^{77}$. The pump design is proved to have comparable washout ( $99.4 \%$ of blood will be washed out after 3 cardiac cycle), and durability ( 5 years) $80-82$. 


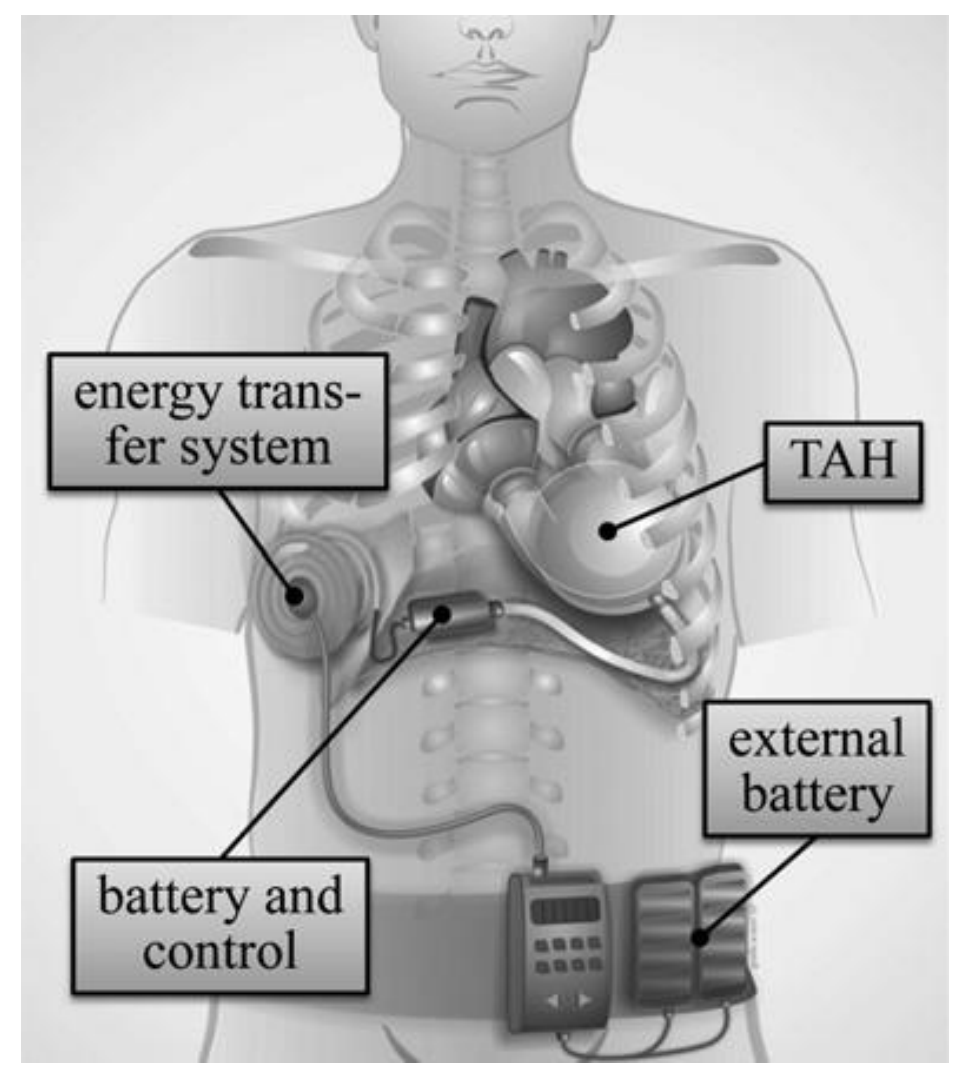

Fig. 6 ReinHeart System

\section{Discussion}

This is the first systemic review on the engineering perspective for pulsatile devices in recent 20 years. In the era of CF-MCSD, the superior outcome has promoted the prevalence of mechanical circulatory support, allowing us to gain insights into clinical care, physiological demands, and the underlying mechanisms for various complications. The benefits of pulsatile flow are getting more understood and pronounced, encouraging more researchers to develop next-generation devices which bring in pulsatility. The effort of bringing proper pulsatility with continuous flow ventricular assist devices has been made to keep the advantage of their compactness. The artificial pulse of HeartMate 3 
provides some pulsatility to blood flow ${ }^{83}$. CFD analysis indicated good washout flow field inside HeartMate 3 pumps with artificial pulse ${ }^{84}$. HVAD Lavare cycle is a speed modulation algorithm, and has shown promising clinical performance with significantly lower stoke, sepsis and right heart failure rate ${ }^{85}$. However, speed modulation of continuous flow blood pumps doesn't generate sufficient pulsatility (Slaughter2014). Furthermore, the drastic acceleration and deceleration will introduce additional shear stress, which may increase blood damage. NIH is highly influenced by the pump design and speed modulation controller. However, continuous flow blood pumps have been optimized to nominal constant flow condition only. Further studies are still needed to investigate the influence of speed modulation on both hydraulic and hemolysis comprehensively.

The design philosophy has altered considerably. The LVADs before 2008 tend to provide full support, which requires a blood sac larger than the native heart. Therefore, the large size and invasive implantation limit their use in wide patient population, not to mention other complications persisting harm the survival and patient quality of life, such as infection and hemocompatibility-related issues. The LionHeart device symbolized an ambitious attempt in solving the complications; however, the failure was quite detrimental. With the knowledge with modern VADs where high unloading may not be preferred in most of the application, the development starts shifting to offer partial or sub-full circulatory support. Furthermore, various advances in engineering fields such as material, electronics, and nano-technology have risen in the past decades. This opens up the room 
for more design possibilities on selection of new pumping mechanisms and system configuration. For example, the NuPulse iVAS serves as an attempt to combined the advantages of IABP and LVAD - to preserve the mobility of the patient in long-term use while keeping a minimally invasive and short implantation procedure.

Since the compromise/balance is always to be made, it is worthy to evaluate different important factors for PF-VAD to facilitate understanding for designers.

\section{Anatomical fitting}

For volume displacement pumps, decreasing pump size and, in the meanwhile, meet the high flow assistance demand of patients is always a problem. As indicated by SHOCK II trial, $90 \%$ of the survivors are NYHA class I/II patients, early engagement of the pump could be one possible solution for decrease flow demands and increase mortality in the meantime.

Although it might be always the smaller the better for VADs, it does not hold true for TAHs. An appropriate size is required to keep the thorax cavity occupied so that the tissue and other organs don't grow or displace into the wrong places. To accommodate patients with small BSA and not eligible with normal TAHs, smaller TAHs have been designed, such as Syncardia TAH ${ }^{66}$, by simply scaling down the pump size. A numerical study of the effect of the size on stress accumulation showed that the platelets in smaller devices will suffer from higher stress accumulation, which could be attributed to reduced size and higher pumping frequencies ${ }^{80}$. 


\section{Wireless technology}

Percutaneous driveline is a shared problem faced by PF-MCSDs and CF-MCSDs, yet it will be much difficult for PF-MCSDs to address due to system design resulted low efficiency. Temptations to eliminate the driveline have been made before 2008. Around 2000, LionHeart ${ }^{19}$, Abiocor ${ }^{59}$, HeartMate $2^{86}$, reopened the TET research. But the technology back then was immature with problems like low long-term reliability, low implantability and skin complications ${ }^{23}$. Waters et al. developed a Free-Range Resonant Electrical Energy Delivery System (FREE-D) for powering left ventricular assist devices 87, 88 , and the feasibility was demonstrated by Agari et al. ${ }^{89}$. However, the FREE-D system is still sensitive to the coil alignment and the environment, the need of battery implantation, and the transmission distance is no more than 0.7 meter ${ }^{89}$. The improvement of the reliability and transfer distance of wireless transfer will make the future of mechanical circulatory support.

\section{Valves, or valve-less}

Artificial valves in the first-generation MCSDs contribute to the major risk of thrombosis of patients (1-2\% per year) even with aggressive anticoagulation therapy ${ }^{90}$. Regions near the valves exist flow abnormality, such as turbulence, stagnation, and recirculation, resulting in blood cells exposed under non-physiological shear stress in various degrees. Thus, mechanical blood trauma can be induced, including hemolysis, thrombosis, and platelet/particle activation or degradation ${ }^{91}$. Using bioprosthetic valves may bring in a 
more physiological flow field, but the high closing pressure shock induces calcification or valve failure within months ${ }^{92}$. To mediate the shock loading, the CARMAT device uses a kind of filled oil behind the pericardial membrane while the ReinHeart pump modulates the drive speed of the piston.

Since valves for blood pumps do not encounter as many anatomical constraints as in the prosthetic valves for valvular diseases, it seems natural to put in more effort on the design. Blood pump valves can resist higher gradients and worse closing volumes and actually moderate leakage. These leads to more work to provide sufficient cardiac output, but for blood pumps power is not an unsolvable problem and the leakage could serve as kind of purge flow for the blood chamber. This is not directly applicable for the inflow valve but certainly the outflow valve. The inflow could have 2 valves, one (could be mechanical) to take up the main closing pressure with leakage and another behind it (could be flexible) to seal and protect the patient.

However, these designs no doubt complicated pump construction. Until a super biocompatible material to come to solve the longevity issue, another innovative solution for the next generation pulsatile blood pumps could be the valveless design. This requires completely re-considering the design (sometimes the pumping principle) at a system level, as seen in the TORVAD device or those counter-pulsating devices employing the native heart valves. 


\section{Blood contacting, or not}

Like other blood-contacting devices, the interface between the device and blood is crucial for the biocompatibility of blood pumps. Most blood pumps have blood in direct contact with artificial material surfaces, so long-term anticoagulation therapy is required. In these devices, the chemical and morphological characteristics of artificial material (biomaterial) surfaces are crucial for improving biocompatibility. Fibrillar textured blood contacting surface had already been used before 2008 on HeartMate I pumps. Various coatings were applied to improve the hemocompatibility. Thanks to the modern computational and experimental fluid dynamics technologies, flow field in the chamber/sac or in the conduits can be comprehensively optimized before the devices are put into resource intensive invivo tests ${ }^{82}$.

On the other hand, possibility to incorporate non-blood-contacting pump is unique for PF-MCSDs. Varies of new designs have been made after 2008, such as the C-pulse devices and the soft robotic sleeve pump. The idea for these non-blood-contacting devices is to completely eliminate the use of life-time anticoagulation medicine and thus improve the quality of life and drug associated complications.

However, these new pumps normally require active pressing on tissues using foreign materials, potentially leading to rejection reaction and immune suppressing intervention. With the suppressed immune system, patients will be more vulnerable to infectious diseases, which could lead to severe medical problems. Constant deforming certain organs may cause irreversible organ damage. Therefore, the biocompatibility of the 
materials and the compressing motions should be carefully optimized to maximize the benefits.

\section{Conclusion}

Notwithstanding the fact that continuous flow blood pumps show better clinical performance than the first generation of pulsatile blood pumps, they still have some adverse effects that may cause clinical problems, which could be addressed by introducing pulsatile flow. Pulsatility control algorithm of CF-MCSDs is being researched, yet the quantity and morphology of the pulsatility is still unknown to provide optimized assistance. PF-MCSDs could produce stronger pulsatility, operate on accordance with the natural heart operation, which provides physiological assistance. The development of new PF-MCSDs should be focused on reducing the pump size, eliminate the need of percutaneous driveline, improve or eliminate the need of prosthetic valve and blood-contacting surfaces. The next generation PF-MCSDs need physiologically and hemodynamically favored assistance mechanism, less or minimally invasive implantation procedure. With the advance of the technology and the emergence of new designs, pulsatile blood pump may stage a comeback and provide better support for heart failure patients. 


\section{Reference}

1. Benjamin EJ, Blaha MJ, Chiuve SE, et al. Heart Disease and Stroke Statistics-2017 Update: A Report From the American Heart Association. Circulation 2017; 135: e146-e603. DOI: 10.1161/CIR.0000000000000485.

2. Murphy C, Zafar H and Sharif F. An updated review of cardiac devices in heart failure. Irish journal of medical science 2017. DOI: 10.1007/s11845-017-1597-9.

3. Kirklin JK, Naftel DC, Kormos RL, et al. Second INTERMACS annual report: more than 1,000 primary left ventricular assist device implants. The Journal of heart and lung transplantation : the official publication of the International Society for Heart Transplantation 2010; 29: 1-10. DOI: 10.1016/j.healun.2009.10.009.

4. Timms D. A review of clinical ventricular assist devices. Medical engineering \& physics 2011; 33: 1041-1047. DOI: 10.1016/j.medengphy.2011.04.010.

5. Kirklin JK, Pagani FD, Kormos RL, et al. Eighth annual INTERMACS report: Special focus on framing the impact of adverse events. The Journal of heart and lung transplantation : the official publication of the International Society for Heart Transplantation 2017; 36: 1080-1086. DOI: 10.1016/j.healun.2017.07.005.

6. Cheng A, Williamitis CA and Slaughter MS. Comparison of continuous-flow and pulsatile-flow left ventricular assist devices: is there an advantage to pulsatility? Annals of cardiothoracic surgery 2014; 3 : 573-581. DOI: 10.3978/j.issn.2225-319X.2014.08.24.

7. Frazier OH. Unforeseen consequences of therapy with continuous-flow pumps. Circ Heart Fail 2010; 3: 647-649. 2010/11/18. DOI: 10.1161/CIRCHEARTFAILURE.110.959023.

8. Soucy KG, Koenig SC, Giridharan GA, et al. Defining pulsatility during continuous-flow ventricular assist device support. The Journal of heart and lung transplantation : the official publication of the International Society for Heart Transplantation 2013; 32: 581-587. DOI: 10.1016/j.healun.2013.02.010.

9. Soucy KG, Giridharan GA, Choi Y, et al. Rotary pump speed modulation for generating pulsatile flow and phasic left ventricular volume unloading in a bovine model of chronic ischemic heart failure. The Journal of heart and lung transplantation : the official publication of the International Society for Heart Transplantation 2015; 34: 122-131. 2014/12/03. DOI: 10.1016/j.healun.2014.09.017.

10. Drews $\mathrm{T}$, Jurmann $\mathrm{M}$, Michael $\mathrm{D}$, et al. Differences in pulsatile and non-pulsatile mechanical circulatory support in long-term use. The Journal of heart and lung transplantation : the official publication of the International Society for Heart Transplantation 2008; 27: 1096-1101. 2008/10/18. DOI: 10.1016/j.healun.2008.07.007.

11. Giridharan GA, Bartoli CR, Spence PA, et al. Counterpulsation with symphony prevents retrograde carotid, aortic, and coronary flows observed with intra-aortic balloon pump support. Artif Organs 2012; 36: 600-606. 2012/05/18. DOI: 10.1111/j.1525-1594.2012.01456.x.

12. John R, Lee S, Eckman P, et al. Right ventricular failure--a continuing problem in patients with left ventricular assist device support. J Cardiovasc Transl Res 2010; 3: 604-611. 2010/09/03. DOI: 10.1007/s12265-010-9216-4.

13. Giridharan GA, Ewert DL, Pantalos GM, et al. Left Ventricular and Myocardial Perfusion Responses to Volume Unloading and Afterload Reduction in a Computer Simulation. ASAIO Journal 2004; 50: $512-$ 518. DOI: 10.1097/01.Mat.0000136513.21369.75.

14. Takeda J. Experimental study on peripheral circulation during extracorporeal circulation. Kyoto 
University, 1960.

15. Barić D. Why pulsatility still matters: a review of current knowledge. Croatian Medical Journal 2014; 55: 609-620. DOI: 10.3325/cmj.2014.55.609.

16. Vetter HO, Kaulbach HG, Schmitz C, et al. Experience with the Novacor left ventricular assist system as a bridge to cardiac transplantation, including the new wearable system. $J$ Thorac Cardiovasc Surg 1995; 109: 74-80.

17. Maher TR, Butler KC, Poirier VL, et al. HeartMate left ventricular assist devices: a multigeneration of implanted blood pumps. Artificial Organs 2001; 25: 422.

18. Frazier $\mathrm{OH}$, Myers TJ and Radovancević B. The HeartMate left ventricular assist system. Overview and 12-year experience. Texas Heart Institute Journal 1998; 25: 265-271.

19. Mehta SM, Jr PW, Rosenberg G, et al. The LionHeart LVD-2000: a completely implanted left ventricular assist device for chronic circulatory support. Annals of Thoracic Surgery 2001; 71: 183-184.

20. El-Banayosy A, Arusoglu L, Kizner L, et al. Preliminary experience with the LionHeart left ventricular assist device in patients with end-stage heart failure. Annals of Thoracic Surgery 2003; 75: 1469-1475.

21. Pennington DG and Lohmann DP. Novacor LVAS Implantation Technique. Operative Techniques in Thoracic \& Cardiovascular Surgery 1999; 4: 318-329.

22. Spanier TB, Chen JM, Oz MC, et al. Time-dependent cellular population of textured-surface left ventricular assist devices contributes to the development of a biphasic systemic procoagulant response $J$ Thorac Cardiovasc Surg 1999; 118: 404-413.

23. Slaughter MS and Myers TJ. Transcutaneous energy transmission for mechanical circulatory support systems: history, current status, and future prospects. Journal of cardiac surgery 2010; 25: 484-489. DOI: 10.1111/j.1540-8191.2010.01074.x.

24. Yu YC, Antaki JF, Boston JR, et al. Mathematical model of pulsatile blood pump for LVAS control. 1997; 6: 3709-3713 vol.3706.

25. Yu YC, Boston JR, Simaan MA, et al. Pressure-volume relationship of a pulsatile blood pump for ventricular assist device development. Asaio Journal 2001; 47: 293-301.

26. Mccarthy PM, Wang N and Vargo R. Preperitoneal insertion of the HeartMate 1000 IP implantable left ventricular assist device. Annals of Thoracic Surgery 1994; 57: 634.

27. Frazier OH, Duncan JM, Radovancevic B, et al. Successful bridge to heart transplantation with a new left ventricular assist device. The Journal of heart and lung transplantation : the official publication of the International Society for Heart Transplantation 1992; 11: 530-537.

28. PGM J, DR W and PM P. Long term home discharge support with novacor LVAS. The Journal of heart and lung transplantation : the official publication of the International Society for Heart Transplantation. 1999, p. 67-68.

29. Long JW. Advanced mechanical circulatory support with the HeartMate left ventricular assist device in the year 2000. Annals of Thoracic Surgery 2001; 71: S176.

30. Pae WE, Connell JM, Adelowo A, et al. Does total implantability reduce infection with the use of a left ventricular assist device? The LionHeart experience in Europe. Journal of Heart \& Lung Transplantation the Official Publication of the International Society for Heart Transplantation 2007; 26: 219.

31. Wang JX, Smith JR and Bonde P. Energy transmission and power sources for mechanical circulatory support devices to achieve total implantability. Annals of Thoracic Surgery 2014; 97: 1467-1474.

32. Slaughter MS. Long-term continuous flow left ventricular assist device support and end-organ 
function: prospects for destination therapy. Journal of cardiac surgery 2010; 25: 490-494.

33. Gohean JR, George MJ, Pate TD, et al. Verification of a computational cardiovascular system model comparing the hemodynamics of a continuous flow to a synchronous valveless pulsatile flow left ventricular assist device. Asaio Journal 2013; 59: 107.

34. Letsou GV, Pate TD, Gohean JR, et al. Improved left ventricular unloading and circulatory support with synchronized pulsatile left ventricular assistance compared with continuous-flow left ventricular assistance in an acute porcine left ventricular failure model. J Thorac Cardiovasc Surg 2010; 140: 11811188.

35. Gohean JR, Larson ER, Hsi BH, et al. Scaling the Low-Shear Pulsatile TORVAD for Pediatric Heart Failure. ASAIO J 2017; 63: 198-206. DOI: 10.1097/MAT.0000000000000460.

36. Roche ET, Horvath MA, Wamala I, et al. Soft robotic sleeve supports heart function. Science Translational Medicine 2017; 9: eaaf3925.

37. Papaioannou TG, Mathioulakis DS, Stamatelopoulos KS, et al. New aspects on the role of blood pressure and arterial stiffness in mechanical assistance by intra-aortic balloon pump: in-vitro data and their application in clinical practice. Artificial Organs 2004; 28: 717.

38. Ferrari G, Khir AW, Fresiello L, et al. Hybrid model analysis of intra-aortic balloon pump performance as a function of ventricular and circulatory parameters. Artificial Organs 2011; 35: 902-911.

39. Meyns BP, Nishimura Y, Jashari R, et al. Ascending versus descending aortic balloon pumping: organ and myocardial perfusion during ischemia. Annals of Thoracic Surgery 2000; 70: 1264-1269.

40. Kapelios CJ, Terrovitis JV, Siskas P, et al. Counterpulsation: A concept with a remarkable past, an established present and a challenging future. International Journal of Cardiology 2014; 172: 318-325.

41. Krishna M and Kai Z. Principles of intra-aortic balloon pump counterpulsation. Continuing Education in Anaesthesia Critical Care \& Pain 2009; 9: 24-28.

42. Slaughter MS, M S, GA G, et al. Counterpulsation devices for myocardial support. Cardiothoracic Surgery Review. 2011.

43. Kolyva C, Pepper JR and Khir AW. Newly Shaped Intra-Aortic Balloons Improve the Performance of Counterpulsation at the Semirecumbent Position: An In Vitro Study. Artif Organs 2016; 40: E146-157. 2016/08/18. DOI: 10.1111/aor.12791.

44. Thiele H, Zeymer U, Neumann FJ, et al. Intraaortic balloon support for myocardial infarction with cardiogenic shock. $N$ Engl J Med 2012; 367: 1287-1296. 2012/08/28. DOI: 10.1056/NEJMoa1208410.

45. Thiele H, Zeymer U, Neumann F-J, et al. Intra-aortic balloon counterpulsation in acute myocardial infarction complicated by cardiogenic shock (IABP-SHOCK II): final 12 month results of a randomised, open-label trial. The Lancet 2013; 382: 1638-1645. DOI: 10.1016/s0140-6736(13)61783-3.

46. Ghia S, Dhawan R, Chaney MA, et al. Can I Go Home With That Balloon Pump? J Cardiothorac Vasc Anesth 2018; 32: 1468-1479. 2018/03/14. DOI: 10.1053/j.jvca.2018.01.021.

47. Jeevanandam V, Song T, Onsager D, et al. The first-in-human experience with a minimally invasive, ambulatory, counterpulsation heart assist system for advanced congestive heart failure. The Journal of heart and lung transplantation : the official publication of the International Society for Heart Transplantation 2018; 37: 1-6. 2017/11/15. DOI: 10.1016/j.healun.2017.10.011.

48. Solanki P. Aortic counterpulsation: C-pulse and other devices for cardiac support. J Cardiovasc Transl Res 2014; 7: 292-300. 2014/02/21. DOI: 10.1007/s12265-014-9548-6.

49. Qureshi MB. Cardiac Output Improvement in Mechanical Circulatory Support Devices. North Dakota State University, 2017. 
50. Jeevanandam VJ, D.; Anderson, A.S.; Martin, S.; Piccione, W.; Heroux, A.L.; Wynne, J.; Stephenson, L.W.; Hsu, J.; Freed, P.S.; Kantrowitz, A. Circulatory Assistance With a Permanent Implantable IABP: Initial Human Experience. Circulation 2002; 106: 183-188. DOI: 10.1161/01.cir.0000032899.55215.44.

51. Black MC, Schumer EM, Rogers M, et al. Sunshine Heart C-Pulse: device for NYHA Class III and ambulatory Class IV heart failure. Future Cardiology 2016; 12: 521-531.

52. Abraham WT, Aggarwal S, Prabhu SD, et al. Ambulatory extra-aortic counterpulsation in patients with moderate to severe chronic heart failure. JACC Heart failure 2014; 2: 526-533. DOI: 10.1016/j.jchf.2014.04.014.

53. Cecere R, Dowling RD and Giannetti N. Initial clinical experience with the Symphony heart assist system. Ann Thorac Surg 2015; 99: 298-301. 2015/01/06. DOI: 10.1016/j.athoracsur.2014.07.094.

54. Warren S, Giridharan GA, Dowling RD, et al. Feasibility of Subcutaneous ECG Leads for Synchronized Timing of a Counterpulsation Device. Cardiovascular Engineering and Technology 2011; 3: 17-25. DOI: 10.1007/s13239-011-0074-2.

55. Malchesky PS. Artificial Organs 2012: a year in review. Artif Organs 2013; 37: 324-349. 2013/03/06. DOI: $10.1111 /$ aor.12057.

56. Dowling RD, Etoch SW, Stevens KA, et al. Current status of the AbioCor implantable replacement heart. Annals of Thoracic Surgery 2001; 71: S147.

57. Dowling RD, Jr GL, Etoch SW, et al. Initial experience with the AbioCor implantable replacement heart system. Journal of Thoracic \& Cardiovascular Surgery 2004; 127: 131-141.

58. Holmes EC. The AbioCor totally implantable replacement heart. Journal of Cardiovascular Nursing 2003; 18: 23.

59. Dowling RD, Gray LAJ, Etoch SW, et al. The AbioCor Implantable Replacement Heart. The Annals of Thaoracic Surgery 2003; 75: 7.

60. Kung RT, Ochs B and Singh PI. A Unique Left-Right Flow Imbalance Compensation Scheme for an Implantable Total Artificial Heart. Asaio Journal 1989; 35: 468-470.

61. Gerosa G, Scuri S, Iop L, et al. Present and future perspectives on total artificial hearts. Annals of cardiothoracic surgery 2014; 3: 595-602. DOI: 10.3978/j.issn.2225-319X.2014.09.05.

62. Sale SM and Smedira NG. Total artificial heart. Best Practice \& Research Clinical Anaesthesiology 2012; 26: 147-165.

63. Ahn JM, Kang DW, Kim HC, et al. In vivo performance evaluation of a transcutaneous energy and information transmission system for the total artificial heart. Asaio Journal 1993; 39: M208-212.

64. Dowling RD, Gray LA, Jr., Etoch SW, et al. Initial experience with the AbioCor implantable replacement heart system. $J$ Thorac Cardiovasc Surg 2004; 127: 131-141. DOI: 10.1016/j.jtcvs.2003.07.023.

65. Slepian MJ, Smith RG and Copel JG. The SynCardia CardioWest ${ }^{\mathrm{TM}}$ Total Artificial Heart. Treatment of Advanced Heart Disease 2006: 473-490.

66. Slepian MJ, Alemu Y, Girdhar G, et al. The Syncardia total artificial heart: in vivo, in vitro, and computational modeling studies. Journal of biomechanics 2013; 46: 266-275. DOI: 10.1016/j.jbiomech.2012.11.032.

67. Gray NA, Jr. and Selzman CH. Current status of the total artificial heart. American heart journal 2006; 152: 4-10. DOI: 10.1016/j.ahj.2005.10.024.

68. Barnard J and Tsui SS. The total artificial heart in a cardiac replacement therapy programme. British Journal of Hospital Medicine 2012; 73: 672-676. 
69. Jaroszewski DE, Anderson EM, Pierce CN, et al. The SynCardia freedom driver: a portable driver for discharge home with the total artificial heart. Journal of Heart \& Lung Transplantation the Official Publication of the International Society for Heart Transplantation 2011; 30: 844-845.

70. Mohacsi P and Leprince P. The CARMAT total artificial heart. European journal of cardio-thoracic surgery: official journal of the European Association for Cardio-thoracic Surgery 2014; 46: 933-934. DOI: 10.1093/ejcts/ezu333.

71. Copeland JG, Smith RG, Arabia FA, et al. Cardiac replacement with a total artificial heart as a bridge to transplantation. New England Journal of Medicine 2004; 351: 859.

72. Samak M, Fatullayev J, Sabashnikov A, et al. Past and Present of Total Artificial Heart Therapy: A Success Story. Medical science monitor basic research 2015; 21: 183-190. DOI: 10.12659/MSMBR.895418.

73. Torregrossa G, Morshuis M, Varghese R, et al. Results with SynCardia total artificial heart beyond 1 year. ASAIO J 2014; 60: 626-634. DOI: 10.1097/MAT.0000000000000132.

74. Latremouille C, Duveau D, Cholley B, et al. Animal studies with the Carmat bioprosthetic total artificial heart. European journal of cardio-thoracic surgery : official journal of the European Association for Cardio-thoracic Surgery 2015; 47: e172-178; discussion e178-179. DOI: 10.1093/ejcts/ezv010.

75. Jansen $\mathrm{P}$, van Oeveren W, Capel A, et al. In vitro haemocompatibility of a novel bioprosthetic total artificial heart. European journal of cardio-thoracic surgery : official journal of the European Association for Cardio-thoracic Surgery 2012; 41: e166-172. DOI: 10.1093/ejcts/ezs187.

76. Koerfer R, Spiliopoulos S, Finocchiaro T, et al. Paving the way for destination therapy of end-stage biventricular heart failure: the ReinHeart total artificial heart concept. European journal of cardio-thoracic surgery : official journal of the European Association for Cardio-thoracic Surgery 2014; 46: 935-936. DOI: 10.1093/ejcts/ezu317.

77. Pelletier B, Spiliopoulos S, Finocchiaro T, et al. System overview of the fully implantable destination therapy--ReinHeart-total artificial heart. European journal of cardio-thoracic surgery : official journal of the European Association for Cardio-thoracic Surgery 2015; 47: 80-86. DOI: 10.1093/ejcts/ezu321.

78. Graf F, Rossbroich R, Finocchiaro T, et al. Investigation of the Durability of a Diaphragm for a Total Artificial Heart. Artif Organs 2016; 40: 1016-1022. DOI: 10.1111/aor.12659.

79. Cuenca-Navalon E, Laumen M, Finocchiaro T, et al. Estimation of Filling and Afterload Conditions by Pump Intrinsic Parameters in a Pulsatile Total Artificial Heart. Artif Organs 2016; 40: 638-644. DOI: 10.1111/aor.12636.

80. Marom G, Chiu WC, Slepian MJ, et al. Numerical model of total artificial heart hemodynamics and the effect of its size on stress accumulation. Conf Proc IEEE Eng Med Biol Soc 2014; 2014: 5651-5654.

81. Schumer EM, Black MC, Monreal G, et al. Left ventricular assist devices: current controversies and future directions. European heart journal 2016; 37: 3434-3439. 2015/11/07. DOI: 10.1093/eurheartj/ehv590.

82. Petukhov DS, Selishchev SV and Telyshev DV. Prospects for Development of Technologies for Complete Replacement of Heart Function by Mechanical Circulatory Support Systems. Biomedical Engineering 2016; 49: 258-262. DOI: 10.1007/s10527-016-9544-z.

83. Essandoh M, Essandoh G, Stallkamp ED, Jr., et al. Spectral Doppler Analysis of the HeartMate 3 Left Ventricular Assist Device Inflow: New Challenges Presented by the Artificial Pulse Technology. $J$ Cardiothorac Vasc Anesth 2018; 32: e4-e5. 2018/08/16. DOI: 10.1053/j.jvca.2018.07.005.

84. Wiegmann L, Thamsen B, de Zelicourt D, et al. Fluid Dynamics in the HeartMate 3: Influence of the 
Artificial Pulse Feature and Residual Cardiac Pulsation. Artif Organs 2019; 43: 363-376. 2018/08/22. DOI: 10.1111/aor.13346.

85. Kumar J, Elhassan A, Dimitrova G, et al. The Lavare Cycle: A Novel Pulsatile Feature of the HVAD Continuous-Flow Left Ventricular Assist Device. J Cardiothorac Vasc Anesth 2019; 33: 1170-1171. 2019/01/12. DOI: 10.1053/j.jvca.2018.11.029.

86. Rintoul TC and Dolgin A. Thoratec Transcutaneous Energy Transformer System: A Review and Update. ASAIO Journal 2004; 50: 397-400. DOI: 10.1097/01.Mat.0000130517.29153.4b.

87. Waters BH, Sample AP, Bonde P, et al. Powering a Ventricular Assist Device (VAD) With the FreeRange Resonant Electrical Energy Delivery (FREE-D) System. Proceedings of the IEEE 2012; 100: 138149. DOI: 10.1109 /jproc.2011.2165309.

88. Waters BH, Smith JR and Bonde P. Innovative Free-range Resonant Electrical Energy Delivery system (FREE-D System) for a ventricular assist device using wireless power. ASAIO $J$ 2014; 60: 31-37. 2013/12/05. DOI: 10.1097/MAT.0000000000000029.

89. Asgari SS and Bonde P. Implantable physiologic controller for left ventricular assist devices with telemetry capability. $J$ Thorac Cardiovasc Surg 2014; 147: 192-202. 2013/11/02. DOI: 10.1016/j.jtcvs.2013.09.012.

90. Bonow RO, Carabello BA, Chatterjee K, et al. 2008 Focused Update Incorporated Into the ACC/AHA 2006 Guidelines for the Management of Patients With Valvular Heart Disease. Journal of the American College of Cardiology 2008; 52: e1-e142.

91. Bluestein D. Research approaches for studying flow-induced thromboembolic complications in blood recirculating devices. Expert Review of Medical Devices 2004; 1: 65-80.

92. Fioretta ES, Dijkman PE, Emmert MY, et al. The future of heart valve replacement: recent developments and translational challenges for heart valve tissue engineering. J Tissue Eng Regen Med 2018; 12: e323-e335. 2016/10/04. DOI: 10.1002/term.2326. 